\title{
Heterotopie und Black Box
}

\section{Versiegelungskontexte der digitalisierten Psychiatrie}

\section{Einleitung}

Die Psych-Landschaft ${ }^{1}$ erstreckt sich längst nicht mehr nur über Nervenkliniken kurz vor den Mauern unserer Städte, ehemalige Klöster, psychotherapeutische Gemeinschaftspraxen, psychiatrische Ambulanzen, forensische oder psychosomatische Kliniken. Fortgesetzt wird eine mediale Psych-Expansion, ${ }^{2}$ die Ensembles aus Beziehungen, Praktiken, Apparaten, Dokumenten und Psych-Artefakten betrifft, die am Tun ${ }^{3}$ der psychischen Erkrankung und Gesundung beteiligt sind. Diese Medialisierung nahm im 19. Jahrhundert unter anderem durch die Anwendung der Fotografie in der Psychiatrie und psychophysischer Vermessungen der Wahrnehmung ihren Anfang.

Inzwischen ist ein neuer, digital attribuierter Raum hergestellt, für den so unterschiedliche Begriffe wie Cyberspace, Infosphäre oder Informationscape $e^{4}$ geprägt wurden. Aus Sicht der User ${ }^{\star}$ innen der digitalisierten Psychiatrie ${ }^{5}$ - ehemals Patient*innen oder Klient ${ }^{\star}$ innen genannt - finden (Selbst-)Behandlungen vermittelt über Monitore ihrer Endgeräte statt. Folgt man den Diskursen der digitalisierten Psych-Landschaft, kann diversen psychischen Leiden durch Anwendung verschiedener Programme vorgebeugt werden. Allein durch Scans und Analyse etwa der Smartphonenutzung seien psychische Erkrankungen diagnostizierbar. ${ }^{6}$ Therapeutische 1:1-Kontakte könnten durch Chats und Videosprechstunden ergänzt oder gar ersetzt werden. Ein Beispiel, das den Umfang der Möglichkeiten einer digitalisierten Psychiatrie zeigt, ist, dass

1 In Anlehnung an die „Psy-Funktion“ bei Castel et al. 1982, 64ff.: „die das Instrumentarium der technisch-produktivistischen Rationalität auf den Menschen anwendet“.

2 Gemeint ist hier der seit dem 19. Jahrhundert steigende Einfluss von Reproduktionsmedien (z. B. Fotografie) und elektronischen Medien (wie im Abschnitt zur Psychophysik ausgeführt). Laut Sybille Krämer bestehen zwei wichtige Effekte von Medien darin, etwas Vorgefundenes ,in neue Zusammenhänge zu übertragen und damit auch anders sehen und/oder anders gebrauchen zu können." Gleichzeitig sind Medienpraktiken auch solche der „Verkörperung“, also der Herstellung oder Manipulation von etwas. Krämer 2003, 84.

3 Vgl. Mol 2017.

4 Lillemose/Krieger 2015; Floridi 2015 [2014]; Rheingold 1985.

5 Gemeint ist hier nicht die Anstaltspsychiatrie im Sinne von Goffmans totaler Institution oder das psychiatrische Krankenhaus, sondern die o.g. Psych-Landschaft, die Psychotherapie explizit mit einschließt. Eine Trennung zwischen Psychotherapie und Psychiatrie wird hier, ganz im Sinne von Dörner et al. 2019, nicht vollzogen, denn psychiatrisches Tun findet an den unterschiedlichsten Orten unter Einbindung unterschiedlichster Handlungsträger^innen statt.

6 DGPPN 2018.

Ә Open Access. (๑) 2020 Eckhard Geitz, publiziert von De Gruyter. (c) BY-NC-ND Dieses Werk ist lizenziert unter der Creative Commons Attribution-NonCommercial-NoDerivatives 4.0 Lizenz.

https://doi.org/10.1515/9783110701319-008 
selbst das persönliche Einnahmeverhalten von Medikamenten inzwischen durch Sensoren erfassbar und somit für Ärztinnen und User`innen unmittelbar sicht- und dokumentierbar ist. ${ }^{7}$

Zur Beschreibung dessen, was unter Digitalisierung im Gesundheitsbereich verstanden werden kann, bietet die Weltgesundheitsorganisation (WHO) folgende Definition an:

eHealth is the cost-effective and secure use of ICT [IKT, Informations- und Kommunikationstechnologie (Anm. E. G.)] in support of health and health-related fields, including health-care services, health surveillance, health literature, and health education, knowledge and research. ${ }^{8}$

Als mHealth versteht die WHO den Einsatz mobiler Geräte zu direkter gesundheitsbezogener Anwendung. ${ }^{9}$ Telemedizin bezeichnet die Interaktion zwischen Patient ${ }^{\star}$ in und Behandler*in über räumliche Distanz mittels IKT. ${ }^{10}$ Diese Interaktion ist der Telepsychiatrie zuzuordnen, sofern sie im Bereich der Psychiatrie stattfindet. e-Mental Health kann als erweiterter Begriff von Telepsychiatrie verstanden werden, unter dem sowohl Anwendungen mit und ohne therapeutische Begleitung subsumiert werden. ${ }^{11}$

Sowohl Psychiatrie als auch (ihre) Digitalisierung sind in gewissem Maße unzugänglich, abgeschlossen oder versiegelt. Während die Geschichte des Wahnsinns eine von Verbannung, Internierung, Absonderung und - seit den 1960er Jahren auch der Wiedereingliederung ist, werden IKT ihrem Wesen nach typischerweise als Black Boxes verstanden, als unsichtbare Technologien, ${ }^{12}$ deren Funktionsprinzipien sich bestenfalls Fachleuten erschließen, aber nicht der Mehrheit ihrer Nutzerinnen.

In diesem Artikel wird mit zwei theoretischen Werkzeugen zur Untersuchung von Kontexten der Versiegelung der digitalisierten Psychiatrie gearbeitet: Vilém Flussers Konzept der Black Box ${ }^{13}$ und Michel Foucaults Begriff der Heterotopie ${ }^{14}$ werden auf die historischen und empirischen Phänomene von Psychophysik und Behaviorismus angewendet und es wird diskutiert, inwiefern sie sich als geeignet erweisen, um Versiegelungskontexte der digitalisierten Psychiatrie zu identifizieren und zu analysieren.

Michels Foucaults Konzeption der Heterotopie und seine Darstellung relevanter Charakteristika solcher Gegenorte sind Ausgangspunkt der Untersuchung. Im zweiten Schritt wird Vilém Flussers Entwurf der Black Box vorgestellt - in seinem Verständnis eine im Wesentlichen kybernetische Apparateprogrammierung. Die zur Beschreibung von Heterotopie und Black Box herausgearbeiteten Begriffe werden

7 https://www.abilifymycite.com/ (Stand: 10.12.2019).

8 WHO 2016, 5.

9 WHO 2016, 27.

10 WHO 2016, 56.

11 Mucic/ Hilty 2016, 4.

12 Vgl. Latour 2002.

13 Flusser 1989.

14 Foucault 2005 [1984]. 
im Weiteren als theoretische Werkzeuge an zwei grundlegenden Knotenpunkten genutzt: am 1879 von Wilhelm Wundt gegründeten Leipziger Institut für experimentelle Psychologie und an der Schnittstelle zwischen Behaviorismus und Kybernetik, wie sie 1943 in „Behavior, Purpose and Teleology“ von Arturo Rosenblueth, Norbert Wiener und Julian Bigelow verzeichnet wurde.

Abschließend wird diskutiert, mit welchen Begriffen sich das Black Box-artige Anderswo der Psychiatrie in ihrer digitalisierten Version verstehen lässt.

\section{Heterotopie}

Die sich ausdifferenzierende Vielgestaltigkeit räumlicher Anordnungen, in denen Gesellschaft seit der Moderne stattfindet, wird durch die Entwicklung von IKT noch komplexer. So stellt sich mit Blick auf das Phänomen der Computerisierung samt ihrer Vernetzung die Frage, wie diese Räume (begrifflich) zu fassen sind. Handelt es sich bei den neu entstehenden digitalen Räumen um beliebig manipulierbare utopische Landschaften? Können sich Bildschirminhalte in alles verwandeln, was die Fantasie von User ${ }^{\star}$ innen zulässt? Eine solch emphatische Sicht ist mit dem von Howard Rheingold geprägten Begriff Informationscape verknüpft, der sich auf die bahnbrechende Entwicklung des augmented window bezieht, eines erweiterten, magischen Fensters, eines Neben- und Auseinanders von Einheiten eines neuen Raums, den Douglas C. Engelbart 1968 während der Fall Joint Computer Conference in San Francisco eröffnete. ${ }^{15}$ Diese neue Virtualität steht am Anfang von inzwischen vollständig in den Alltag integrierten und stets verfügbaren Miniaturen der Gegenwart.

Seit der amerikanische Schriftsteller William Gibson 1982 die Kurzgeschichte Burning Chrome veröffentlichte, wurde der Begriff des Cyberspace immer populärer, um vereinnahmende Effekte von Verdatung zu beschreiben.

A graphic representation of data abstracted from banks of every computer in the human system, the concept has been associated with notions of an all-encompassing digitisation of our existence, perception and awareness, often with dark socio-political overtones. ${ }^{16}$

15 Präsentiert wurden „Fenster“ auf einem Bildschirm, gesteuert von einer ebenfalls neuesten Entwicklung, der Maus. Die heute so selbstverständlich zur informationellen Wirklichkeit gehörende Symbolverarbeitung, glich laut Rheingold einer fiktionalen Reise in bis dato ungekannte Welten: „The symbolic domain, from minutiae to the grandest features, could be rearranged at will by the informationaut, who watched through his window while he navigated his vehicle and the audience witnessed it all on the big screen. Informational features were reordered, juxtaposed, deleted, nested, linked, chained, subdivided, inserted, revised, referenced, expanded, summarized - all with fingertip commands." Rheingold 1985, 190-191.

16 Lillemose/Kryger 2015. 
Erstmalig verwendet wurde der Begriff jedoch bereits zehn Jahre vor seiner Popularisierung als Name für ein Atelier, in dem es darum ging, über Design-Möglichkeiten und Wirkungen im Zeitalter der Kybernetik zu reflektieren. ${ }^{17}$ Zugespitzt wird das Prinzip der Vereinnahmung durch umfangreiche (Selbst-)verdatung und das Internet der Dinge bei Luciano Floridi, der meint, weit gefasst sei die Infosphäre „ein Begriff, der sich synonym mit Wirklichkeit verwenden lässt, wenn wir Letztere informationell auffassen. In diesem Fall ließe sich sagen, was wirklich ist, ist informationell, und was informationell ist, ist wirklich."18

Um die Andersartigkeit des vernetzten Raums in den Blick zu nehmen, der als Informationscape, Cyberspace, Infosphäre oder digitaler Raum gefasst werden kann, scheint Michel Foucaults Konzept der Heterotopie als theoretisches Werkzeug geeignet. „Wir leben“, formuliert Foucault 1967 „im Zeitalter der Gleichzeitigkeit, des Aneinanderreihens, des Nahen und des Fernen, des Nebeneinander und des Zerstreuten. Die Welt wird heute nicht so sehr als ein großes Lebewesen verstanden, das sich in der Zeit entwickelt, sondern als ein Netz, dessen Stränge sich kreuzen und Punkte verbinden.“19

Diese Netzwerkhaftigkeit meint Foucault gegenwartsdiagnostisch und bezieht sie nur an einer Stelle - und zwar eher beiläufig - auf moderne IKT. ${ }^{20}$ Entscheidend für Foucaults Begriff der Heterotopie ist, dass sie - ganz im Gegensatz zur Utopie - im Hier und Jetzt zu verorten ist. Laut erstem Grundsatz über Heterotopien gehören in den Bereich des Anderswo der Abweichungsheterotopie etwa Sanatorien, psychiatrische Anstalten, Gefängnisse und Altersheime, also Räume sowohl zur Versammlung von Abweichungen und Abweichler^innen als auch zu ihrer Korrektur. Zweitens sei es für Heterotopien charakteristisch, dass die Räume ihrer Funktion nach Transformationsprozessen unterzogen seien. Zwar führt Foucault als Beispiel für Funktionswandel die Ortsverlagerungen von Friedhöfen in den Städten des 19. Jahrhunderts auf, doch würden sich zur Plausibilisierung des Funktionswandels auch Anstaltsorte eignen, beispielsweise mittelalterliche Klöster nach der Reformation. Das Kloster Breitenau in Hessen steht exemplarisch für einen solchen Funktionswandel. In

17 Lillemose/Kryger 2015.

18 Floridi 2015 [2014], 64.

19 Foucault 2005 [1984], 931. Ausführlich zur Genese von Foucaults Begriff der Heterotopie: Chlada 2005.

20 „Heute tritt die Lage an die Stelle der Ausdehnung, welche einst die Lokalisierung ersetzte. Die Lage wird bestimmt durch Nachbarschaftsbeziehungen zwischen Punkten oder Elementen, die man formal als mathematische Reihen, Bäume oder Gitter beschreiben kann. Andererseits wissen wir, welche Bedeutung Probleme der Lage oder der Platzierung in der heutigen Technik haben. Man denke etwa an die Speicherung von Information oder von Teilergebnissen einer Rechnung im Speicher einer Maschine, an die zufallsbestimmte Zirkulation diskreter Elemente (zum Beispiel von Autos im Straßenverkehr oder von Tönen in einer Telefonleitung), an das Auffinden markierter oder codierter Elemente innerhalb einer Menge, die entweder eine Zufallsverteilung aufweist oder durch eindeutige bzw. mehrdeutige Zuordnung geordnet ist.“ Foucault 2005 [1984], 932-933. 
den Jahrhunderten nach seiner Auflösung als Schafstall, Kirche, Arbeitshaus, Konzentrationslager, Arbeitslager und Mädchenheim genutzt, werden die letzten psychiatrischen Einrichtungen auf dem Klostergelände gegenwärtig geschlossen. Drittens zeichne Heterotopien ihre Multiplizität aus, ihre Vielgestaltigkeit. Foucault nennt als Beispiel das Kino, auf dessen zweidimensionale Leinwand ein dreidimensionaler Raum projiziert wird. Viertens gebe es etwas wie Heterotopien der Zeit - Orte, wo Zeit gesammelt wird - wie in einem Archiv oder einem Museum - und Orte, wo Zeit sich verflüchtigt - wie auf Festen oder Jahrmärkten. Fünftens seien Heterotopien durch Systeme von Öffnungen und Schließungen charakterisiert. Zugang sei an bestimmte Konventionen geknüpft, wie - laut Foucault - das Ablegen der Kleidung im Falle der Sauna. Schließlich zeichne es Heterotopien aus, dass sie zwischen den Polen des illusionären und des realen Raums changieren, dass also der illusionäre Raum gegen den realen Raum in Stellung gebracht wird und ihn unterminiert. Foucault führt dafür das Beispiel der Freudenhäuser auf. ${ }^{21}$

Die Erfordernisse zur Hervorbringung von Heterotopien liegen in Krisen oder Abweichungen. Sie sind Orte des Formens, des Geformtwerdens und des Sichformens. Insofern ähneln sie dem Dispositiv-Konzept. Die netzwerkartigen Verknüpfungen von Dispositiven - verstanden als strategische Antworten auf gesellschaftliche Notstände sind Verknüpfungen von Praktiken und Beziehungen. Die netzwerkartigen Verknüpfungen von Heterotopien sind solche von Räumen. ${ }^{22}$ Mit dem Heterotopie-Konzept, so die vorläufige Annahme, lassen sich also die vielgestaltigen Verknüpfungen und multiplen Räume der digitalisierten Psychiatrie analytisch aufschließen/aufschlüsseln.

\section{Black Boxes}

„Wer oder was wurde in der Salpetrière den photographischen Bildern dienstbar gemacht?“ ${ }^{23}$ Diese Gretchenfrage hinsichtlich des Anteils der Fotografie an der Herstellung einer psychischen Erkrankung stellt Georges Didi-Huberman in seiner umfangreichen Studie Erfindung der Hysterie. Ein Gegenstand der Untersuchung ist der Einfluss der damals noch revolutionären Technologie auf psychiatrische Diagnostik und Wissensproduktion in der Pariser Salpetrière, eine „Art weiblicher Hölle, eine città dolorosa, viertausend Frauen, unheilbare oder verrückte, waren in sie eingeschlossen. Ein Albtraum in Paris, in nächster Nähe zu seiner ,Belle Epoque““.24 Die Fotografie war ab dem zweiten Drittel des 19. Jahrhunderts vollständig in die klinische Praxis der Salpetrière integriert, ein voll ausgestattetes Fotoatelier war eingerichtet,

21 Vgl. Ziemann 2017.

22 Vgl. Foucault 1978, $120 \mathrm{ff}$.

23 Didi-Huberman 1997, 58.

24 Didi-Huberman 1997, 8. 
inklusive einer Kamera mit Zeitschaltung und 12 Objektiven, die dazu dienten, einen „großen, vollständigen und regelmäßigen hysterischen“ Anfall aufzunehmen und zur Grundlage der Typisierung von Hysterie zu machen. ${ }^{25}$ Das Black Box-artige psychiatrische Anderswo war fortan auch jenseits der Salpetrière mit dem Fotoapparat, der Black Box per se, verschränkt. ${ }^{26}$ Die von Didi-Huberman aufgeworfene Frage, wer oder was in der Psychiatrie der Funktion der Fotografie unterworfen sei, führt zu Vilém Flussers Essay Für eine Philosophie der Fotografie, die durchaus auch als Philosophie seines Begriffs von Black Box gelesen werden kann. ${ }^{27}$ Flusser versteht das Aufkommen der Fotografie im 19. Jahrhundert als Zeitenwechsel hinsichtlich der Art und Weise, wie Menschen kommunizieren, als einen Übergang von Schrift zu Bild. Der informationelle Umbruch, von ihm als „Universum der Technischen Bilder“ bezeichnet, setzt laut Flusser früher an als in den Konzepten der Informationscape, des Cyberspace und der Infosphäre. ${ }^{28}$ Entscheidend sei, dass „die ,Objektivität‘ der technischen Bilder eine Täuschung ist“", dass hier also tendenziell das Risiko eines Auflösens der Unterscheidung zwischen Abbild und Abgebildetem liege. ${ }^{29}$ Das traditionelle, das gemalte Bild sei noch relativ leicht zu decodieren. Im Wesentlichen gehe es darum, die Absicht des Malers zu entschlüsseln. Dies sei bei technischen Bildern deutlich schwieriger:

Zwar schiebt sich auch bei ihnen ein Faktor zwischen sie und ihre Bedeutung, nämlich eine Kamera und ein sie bedienender Mensch (zum Beispiel ein Fotograf), aber es sieht nicht so aus, als würde dieser Komplex ,Apparat/Operator ' die Kette zwischen Bild und Bedeutung unterbrechen. Im Gegenteil: Die Bedeutung scheint auf der einen Seite (Input) hineinzufließen, um auf der anderen Seite (Output) wieder herauszufließen, wobei der Ablauf selbst, das Geschehen innerhalb des Komplexes verborgen bleibt: eine ,Black Box‘ also. ${ }^{30}$

Zur Entlarvung der Täuschung durch technische Bilder ist der Blick in das Innere der Black Box, des Apparates notwendig, wofür Flusser theoretisches Werkzeug in Form der Fotometaphorik bereit hält. Eine Antwort auf die Frage, wer der Fotografie, den technischen Bildern, dienstbar gemacht wird, zielt Flusser auf die Verschmelzung von Apparat und Mensch ab:

\footnotetext{
Anders als der vom Werkzeug umgebene Handwerker und der an der Maschine stehende Arbeiter ist der Fotograf im Apparat drinnen und mit dem Apparat verflochten. Dies ist eine neuartige Funktion, bei der der Mensch weder die Konstante noch die Variable ist, sondern in der Mensch und Apparat zur Einheit verschwimmen. ${ }^{31}$
}

25 Didi-Hubermann 1997, 132-133.

26 Zu Medien und Psychiatrie vgl.: Stingelin 1988; Regener 1999; Regener 2010.

27 Flusser 1989.

28 Flusser 1989, 65.

29 Flusser 1989, 14.

30 Flusser 1989, 26.

31 Flusser 1989, 25-26. 
Diese Verschmelzung liegt vor allem in der Mannigfaltigkeit der Black Box-Programme begründet, die User`innen auf der Suche nach immer neuem Output immer neue Inputs eingeben lässt; ein unabschließbarer Vorgang. Die Inputs können als Einstellungen zweiter Ordnung aufgefasst werden, da ein Apparat nur insoweit eingestellt werden kann, wie es bereits durchgeführte Voreinstellungen seitens des Apparateherstellers zulassen. Flusser bringt diesen Umstand auf die knappe Formel, Schwarz und Weiß seien theoretische Begriffe. Dies gilt grundsätzlich für alle Farben. So bestimmt die Herstellerdefinition etwa der Farbe Gelb, in welchem Gelb ein abfotografiertes gelbes T-Shirt bei gleichen sekundären Einstellungen abgebildet werden kann. Im Wesentlichen hält Flusser das Fotouniversum seit Beginn seiner Entwicklung für digital - in seiner Sprache: für „mosaikförmig“32 -, da die Fotoproduktion darauf hinauslaufe, einzelne Bildpunkte zu programmieren, aus denen dann die Fotografie entstehe. Diese entspreche der Vergegenständlichung einer Assemblage aus Apparat, Motiv, Fotograf, Chemie und Einstellungen.

Eine relevante Folge der scheinbaren Objektivität technischer Bilder ist somit, dass sie Modell für Einstellungen und Verhalten werden. Insofern werden ihnen gleichfalls die Betrachterinnen dienstbar gemacht. Was von der Bildebene ausgeht, fasst Flusser im Kern als kybernetische Steuerung auf: Betrachter^innen bekommen Feedbacks, erleben, erkennen und werten „die Welt in Funktion von Fotos“. ${ }^{33}$ Kritisch gesehen heißt das für Flusser:

Nun ist diese Art von Dasein, in dem alles Erfahren, Erkennen, Werten und Handeln in punktartige Elemente (in ,bits') zerlegt werden kann, bekannt: Es ist das Dasein von Robotern. Das Fotouniversum und alle apparatischen Universen robotisieren den Menschen und die Gesellschaft. ${ }^{34}$

Mit allen apparatischen Universen meint Flusser weitere Black Boxes, die nach dem Prinzip der Ur-Black Box, dem Fotoapparat funktionieren. Diese verschränkten Black Boxes, Super-Black Boxes, seien ein Charakteristikum der postindustriellen Gesellschaft, die im Modus dieser Apparate eingestellt wird. In Bezug auf die digitalisierte Version der Psychiatrie wäre mit SuperBlack Box etwa eine Verschränkung aus Geräten, Programmen, Institutionen, User^innen samt ihrer Praktiken gemeint.

Während Foucaults Begriff der „Kritik“ auf die Rekonstruktion von Herstellungsprozessen, ihrer Genealogie und ihrer Folgen hinausläuft, zielt Flussers Begriff einer „kritischen Praxis“ auf die Rekonstruktion von (Apparat-)Einstellungen. In den folgenden Abschnitten wird gezeigt, inwiefern sich diese beiden Ansätze für die Rekonstruktion von Versiegelungskontexten der digitalen Psychiatrie aufeinander beziehen lassen.

32 Flusser 1989, 64.

33 Flusser 1989.

34 Flusser 1989. 


\title{
Psychophysik, Behaviorismus, Kybernetik
}

\begin{abstract}
Erst nachdem Wahrnehmungsprozesse so kalt und unmenschlich erforscht waren, als wären sie technische Medien, stand der Konstruktion wirklicher Medien, die diese selbe Wahrnehmung täuschen und/oder simulieren können, nichts mehr im Weg. ${ }^{35}$
\end{abstract}

Als prototypisch für den hier von Friedrich Kittler beschriebenen Raum, in dem sich Voraussetzungen für die Herstellung „wirklicher Medien“ wie Telefon, Grammofon und Phonograph manifestierten, kann das Institut für experimentelle Psychologie in Leipzig gelten. In der Psychologie des 19. Jahrhunderts hatte sich ihr Gegenstandsbereich vom Ausdeuten der Seele hin zur Messung empirisch erfassbaren Einwirkens auf das Bewusstsein verschoben. Der Gründer des Leipziger Instituts, Wilhelm Wundt, begriff diese Verschiebung als Streben nach dem naturwissenschaftlichen Vorbild. ${ }^{36}$ Auf diesem Weg der Emanzipation der Psychologie als eigenständige (Natur-)Wissenschaft folgte Wundt seinen Zeitgenossen Hermann von Helmholtz und Gustav Theodor Fechner. Helmholtz hatte die Wechselwirkung zwischen Wahrnehmung und Empfindung untersucht - etwa beim Farben- und Tiefensehen, ${ }^{37}$ Fechner initiierte durch seine Psychophysik ${ }^{38}$ Verfahren zum Messen der Empfindung. ${ }^{39}$ Das Institut für experimentelle Psychologie in Leipzig wurde der Ort für umfangreiches Vermessen der Wahrnehmung. Anlässlich einer Festrede zum 500-jährigen Bestehen der Universität Leipzig stellte Wilhelm Wundt 1909 den Ehrengästen das Institut und seine 30-jährige Geschichte vor. ${ }^{40}$ Der Gründer und Leiter des Instituts gab einen detaillierten Bericht über bauliche Bedingungen, Ausstattung der Räume, besondere Messinstrumente sowie Arbeitsabläufe. Wundt beschreibt die räumliche Anordnung des Instituts und die damit in Verbindung stehenden Praktiken. Im „Außen“ entsteht ein netzwerkartig strukturierter Raum, der das Innen dekodieren soll:

Es bleibt schließlich noch zu bemerken, daß alle Räume des Instituts, die zu experimentellen Arbeiten dienen, untereinander sowohl mit Leitungen für Starkstrom, wie mit solchen für Akkumulatoren und für die Meidinger-Batterie in Nr. 6 versehen sind. Eine solche allseitige Verbindung zum Behuf der Zuführung von Strömen und Kraftquellen, wie von Signalen und Telephonleitungen ist durch die besonderen Bedingungen des psychologischen Experiments geboten, da dieses für viele Zwecke das Arbeiten der verschiedenen Teilnehmer einer Versuchsgruppe in getrennten Räumen erforderlich macht. Ebenso ist in diesen Bedingungen die Teilung des Laboratoriums in eine verhältnismäßig große Zahl kleinerer Zimmer begründet, neben denen nur wenige größere für spezielle räumlich ausgedehnte Versuchsanordnungen nötig sind. Überdies

35 Kittler 1997, 86.

36 Wundt 1983a, 2.

37 Von Helmholtz 1856; Rechenberger 1994.

38 Fechner 1860.

39 Eckart 2010, $56 \mathrm{ff}$.

40 Wundt 1983b; vgl. zur Geschichte des Instituts Lamberti 1995. 
ist ein Zusammenarbeiten mehrerer unabhängiger Beobachter in einem und demselben Raum, wie ein solches in physikalischen und chemischen Laboratorien in der Regel stattfinden kann, hier durchgehend ausgeschlossen. ${ }^{41}$

Der Ort beinhaltete nicht nur Instrumente zur Quantifizierung des Tastens, des Hörens, des Riechens, des Schmeckens, des Sehens, des Zeitwahrnehmens, des Raumwahrnehmens, der Aufmerksamkeit und des Gefühls. ${ }^{42}$ Auch das Gebäude selbst diente dem Experiment. Das Labor war eine Art programmierter Apparat mit dicken Wänden und Matratzentüren zum Schallschutz, einem 37,10 m langen Flur, Dunkelkammern, einem von Elektromotoren bewegten Vorhang zum Verdunkeln der Oberlichter im Vorlesungssaal und genau angeordneten Arbeits- und Vorlesungsräumen. Zum Messarsenal gehörten unter anderem Fallphonometer, Photometer, Druckwaagen für Tastversuche, Stimmgabelserien, Tonmesser, Obertonapparate, Apparate zur Sonderung und zur Mischung der Farben des prismischen Spektrums und Apparate zur grafischen Registrierung von Puls, Atmung und Volumenschwankungen infolge veränderter Blutgefäßinnervation. ${ }^{43}$ Die Prämisse aller Messungen war, über die Kontrolle der Umweltreize und ihre gezielte und präzise Setzung Aufschluss über innere Vorgänge, nämlich solche der Seele bekommen zu können:

\begin{abstract}
Es wird eben auch festgestellt das Verhalten der Seele zu den äußeren Einwirkungen, und indem man diese variirt, gelangt man zu den Gesetzen, denen das psychische Leben als solches unterworfen ist. Die Sinnesreize sind um es kurz auszudrücken, für uns nichts anderes als experimentelle Hülfsmittel. Indem wir die Sinnesreize mannichfach verändern und dabei fortwährend die psychischen Erscheinungen studiren, bringen wir nur das Prinzip zur Anwendung, in welchem das Wesen der experimentellen Methode besteht, „wir verändern, um mit Baco zu reden, die Umstände, unter welchen Erscheinungen auftreten.“
\end{abstract}

Die Netzwerkartigkeit der Apparateanordnung lässt sich als Vorwegnahme des Internets der Dinge lesen, die schon ganz zu Beginn der Medienepoche der elektronischen Medien möglichst diskrete Daten erfasste und miteinander verband. Das Leipziger Institut war beides: Heterotopie und Black Box. Es wurde insofern zu einem Gegenort, als sich hier die experimentelle Psychologie - ganz nach dem Anspruch von Wundt - als Naturwissenschaft emanzipieren konnte. Damit wurde es zu einem Ort umfangreicher Wahrnehmungsvermessung und gleichzeitig auch der Vermessung von Abweichungen. In Funktion der Vermessung überlagerten sich Räume. Es wurden Einstellungen erster und zweiter Ordnung programmiert und gemessen. Der Apparat Institut war eine mit weiteren Apparaten verschränkte SuperBlack Box und die entstehende Einheit, in der Apparat und Mensch miteinander verschmolzen, kann

41 Wundt 1983b; diverse Artikel zu Messverfahren und den konkreten Abläufen in: Schumann 2004.

42 Wundt 1983b, 525.

43 Wundt 1983b, 519. 
als Herstellungsprozess der modernen Psychologie verstanden werden, die ohne programmierte Messapparate nicht denkbar wäre.

Die experimentelle Psychologie und Wundts Institut waren international und besonders in den Vereinigten Staaten sehr einflussreich. So wurde Wundts Labor etwa an diversen amerikanischen Universitäten nachgebaut. Doch trotz aller Arbeit an der Vermessung der Psyche und trotz des entscheidenden Beitrags zur Etablierung der Psychologie als eigenständige Wissenschaft verlor die experimentelle Psychologie zu Beginn des 20 Jahrhunderts gegenüber der neu aufkommenden Strömung des Behaviorismus an Bedeutung. ${ }^{44}$ Zwar beanspruchte die experimentelle Psychologie für ihre Forschung Objektivität, doch genau diese sprach der Behaviorismus ihr ab. ${ }^{45}$ Gegenstand der Vermessung ist für den Behaviorismus nicht das Bewusstsein, sondern das Verhalten von Versuchstieren und Proband ${ }^{\star}$ innen. ${ }^{46}$ Die Prämisse war nicht nur die prinzipielle Vorhersagbarkeit von Verhalten, sondern gar die Programmierung von Kindern, die bei entsprechender Manipulation der Umweltreize in jedem nur denkbaren Beruf zu Spezialisten werden könnten. John B. Watson betont in seiner behavioristischen Programmschrift den Vorzug der praktischen Anwendbarkeit der Psychologie, wie er sie versteht. ${ }^{47}$ Damit verschiebt sich nicht nur der Gegenstandsbereich dessen, was im psychologischen Labor gemessen wird, sondern auch der Ort der Psychologie selbst. Der Begriff „Umwelt“ bezieht sich nun nicht mehr nur auf die Bedingungen im Messraum, sondern auf die Umgebung des Menschen ganz allgemein. Diese formbare Umgebung ist als Arrangement von Reizen operationalisierbar und formt ihrerseits die Praktiken derer, die auf diese Reize reagieren. Anders gesagt: Es braucht keine Apparateverschränkung mehr, keine zu programmierende Black Box, auf die Menschen dann reagieren, sondern Menschen werden selbst zur Black Box, die aufgrund bestimmter Inputs bestimmte Outputs generieren.

Eine abermalige und ausgesprochen folgenreiche Verschiebung des Gegenstands der (behavioristischen) Psychologie und Entstehungsbedingung für die Kybernetik markiert das 1943 von Arturo Rosenblueth, Norbert Wiener und Julian Bigelow verfasste

44 Friedrich et al 1979, $52 \mathrm{ff}$.

45 „Objektivität war eines der Kriterien Wundts für das Experiment, und die Methode der Introspektion bestand ja gerade im Versuch, die subjektiven Äußerungen der ,Versuchspersonen“ zu verobjektivieren, indem man versuchte, die experimentellen Bedingungen, unter denen die Versuchspersonen zur Mitteilung über ihre Empfindungen und Vorstellungen veanlasst wurden, zu verobjektivieren, d. h. wiederholbar zu machen.“ Bruder 1982, 24.

46 Mills 1998; John Mills gibt in seiner Arbeit u. a. einen Überblick über diverse Richtungen des Behaviorismus.

47 „Gebt mir ein Dutzend gesunder, wohlgebildeter Kinder und meine eigene Umwelt, in der ich sie erziehe, und ich garantiere, daß ich jedes nach dem Zufall auswähle und es zu einem Spezialisten in irgendeinem Beruf erziehe, zum Arzt, Richter, Künstler, Kaufmann oder zum Bettler und Dieb, ohne Rücksicht auf seine Begabungen, Neigungen, Fähigkeiten, Anlagen und die Herkunft seiner Vorfahren. [...] Persönlichkeit ist nichts anderes als das Endprodukt unserer Gewohnheitssysteme.“ Watson 1968 [1923], 123. 
Papier „Behavior, Purpose and Teleology“. Rosenblueth et al. legten in ihrer Untersuchung die begrifflichen Grundlagen dafür, das Konzept Behavior sowohl auf Menschen als auch auf Maschinen anzuwenden - Maschinen wurden also selbst zum Gegenstand der behavioristischen Psychologie. ${ }^{48}$ Wie sie selbst ausführten, verfolgten sie dazu die Absicht, zu klären, was unter behavioristischer Untersuchung von natural events zu verstehen sei und wie der Begriff Behavior klassifiziert werden könne. Wie die Behavioristen des frühen 20. Jahrhunderts interessierten sie sich für die Beziehungen zwischen Input und Output und übergingen in ihrer Analyse die innere Struktur der zu untersuchenden Gegenstände. Bei Mensch und Maschine interessierten sie sich für Output, der nicht eine direkte Übersetzung der „Input-Energie“ war, sondern für Output, der im Inneren der (menschlichen und maschinenhaften) Black Box verändert wurde (Active Behavior). Zentral war für Rosenblueth et al., dass das Verhalten von Mensch und Maschine zielgerichtet sein kann und dass die Absicht des Verhaltens durch Feedback ${ }^{49}$ kontrolliert wird - womit sie den Begriff Teleologie bestimmen. Das Ziel dieses Konzepts besteht in der Vorhersagbarkeit feedbackgesteuerten, zielgerichteten Verhaltens und der in den 1940er Jahren kriegsbedingte Zweck war die Flugabwehr. Im Kapitel Kybernetik und Psychopathologie aus seiner 1948 erschienenen Schrift Kybernetik bezieht Wiener ausführlich Schlüsse aus der Nachrichtentechnik und der Maschinensteuerung auf psychische Störungen und argumentiert, dass gegenseitige Bezüge zum Verständnis beider Phänomene geeignet seien. ${ }^{50}$ Die Wirksamkeit, Wechselseitigkeit und Wirkmächtigkeit kybernetischer Steuerung veränderte sich in ihrer Qualität durch die Einführung von Computerbildschirmen als Feedbackoberfläche, ${ }^{51}$ die neben allen Möglichkeiten von Kommunikation und (Selbst-)Behandlung der Gegenwart auch die psychiatrische bedingt.

\section{Digitalisierte Psychiatrie}

Die Landschaft der digitalisierten Psychiatrie ist nicht minder komplex und verschachtelt als die Psych-Landschaft vor ihrer Digitalisierung. Es handelt sich dabei um ein Netzwerk aus verschiedensten Institutionen, Programmen, Apparaten,

48 Rosenblueth/Wiener/Bigelow 1943.

49 Ausführlich zur Bedeutung von Feedback in Psychologie, Psychotherapie und Unternehmenskommunikation: Bröckling 2008, 347: „Sich selbst, ein Unternehmen oder die Gesellschaft als ganze zu lenken heißt mehr denn je, Rückkopplungsschleifen $\mathrm{zu}$ installieren. Vielleicht finden sich die Schaltpläne jener machine à gouverner, von der die frühen Kybernetiker träumten, gar nicht auf den Platinen der Computer, sondern in den Trainingsmanualen der Gruppendynamiker und all den $\mathrm{Zu}$ friedenheitsbarometern, Evaluationsberichten und Rankinglisten, mit denen Evaluationsspezialisten uns tagtäglich traktieren.“

50 Wiener 1963 [1948], 207-222. Canguilhem 2013 [1943], 271ff. greift Wieners Argument in der 1966 erschienenen Neuauflage von Le normal et le pathologique auf. Kritisch dazu: Link 2006, 113.

51 Licklider 1973; Turkle 1984; Krassmann 2013. 
Diskursen, Praktiken und User^innen. Dazu zählen auch - wie im vorangegangenen Abschnitt vorgestellt - ihre technologischen Hervorbringungsbedingungen. Eine analytische Aufschlüsselung der vielgestaltigen Verknüpfungen und multiplen Räume der digitalisierten Psychiatrie kann - folgt man Vilém Flussers Philosophie der Black Box - beim technischen Bild, der Feedbackoberfläche, dem Monitor beginnen. Zugänge zur digitalisierten Psychiatrie führen in aller Regel über Bildschirme von Computern oder den unterschiedlichsten Formen mobiler Endgeräte mit Internetzugang. Mobile Endgeräte können privat genutzte Smartphones oder Medizinprodukte sein, sie können sich in Arztpraxen, Krankenhäusern oder überall dort befinden, wo User`innen auf sie zugreifen. Die über die Endgeräte ansteuerbaren digitalen Räume können von Krankenkassen zur Verfügung gestellt werden oder sie können von Online-(Gesundheits-)Unternehmen kostenlos oder kostenpflichtig angeboten werden, wobei kostenlose Angebote in aller Regel mit der kommerziellen Nutzung von User*innen-Daten einhergehen. Die über den Bildschirm ansteuerbaren Orte werden meist als Smartphone-, Messenger- oder Webapps dargestellt - seien es Kanäle für telepsychiatrische Sprechstunden, diagnostische Programme oder Programme der (Selbst-)Behandlung. Die Websites oder Apps können entweder unmittelbar dem Bereich der digitalisierten Psychiatrie zugerechnet werden, nämlich dann, wenn ihre Hauptfunktion in der psychiatrischen Internetintervention besteht. Oder es handelt sich dabei um kommerzielle Messenger-Dienste, die therapeutisch genutzt werden. In der Architektur digitaler psychiatrischer Räume vergegenständlichen sich unter anderem Diskurse der gesundheitspolitischen Versorgung - der Mangel an Behandlungsplätzen soll kompensiert werden, auf zeitliche Flexibilitätsansprüche der User^innen soll reagiert werden, räumliche Distanzen sollen überwunden werden. Aushandlungsprozesse zwischen (Berufs-)Verbänden, Gesetzgebern, Krankenkassen oder Anbieter^innen von digitalen Produkten haben ebenfalls architektonische Resonanzen - etwa mit der Konsequenz, dass bestimmte Websites oder Apps (nur) unter bestimmten Bedingungen als Medizinprodukt zertifiziert werden müssen. Insbesondere die Expertise von Softwareentwickler`innen ist grundlegend für die Gestaltung jedes Raums der digitalisierten Psychiatrie - notwendigerweise synchronisiert durch die Expertise von psychotherapeutischem Fachpersonal, eingebettet in die spezifischen Bedingungen, unter denen eine spezifische Konfiguration der digitalen Psychiatrie zustande kommt. Die Smartphone-App hat der Psychiatrie zu dienen. Gleichzeitig muss der Hersteller damit auch in der Wertsphäre der Ökonomie bestehen können und gesetzlichen Vorgaben genüge tun. Diese grobe Skizze der SuperBlack Box der digitalisierten Psychiatrie zeigt schematisch, wie vielfältig miteinander verflochten ihre einzelnen Black Boxes sind. Es wird deutlich, wie an jedem Knotenpunkt des Netzwerks ein Komplex aus (Apparate-)Einstellungen entsteht, der Ähnlichkeiten mit dem Komplex aus Fotograf*in-Apparat-Motiv aufweist. Der Unterschied zum von Didi-Hubermann beschriebenen Modus, in dem die Hysterie erfunden wurde, besteht allerdings darin, dass die Bildfläche von mehreren Seiten angesteuert und manipuliert werden kann: von der therapeutischen Seite, von der User`innenseite, von der 
technischen und wirtschaftlichen Seite. Die Frage danach, wer oder was den technischen Bildern, diesen Feedbackoberflächen, in einer digitalisierten Psychiatrie dienstbar gemacht wird, ist also an jedem der hier skizzierten Netzwerkknotenpunkte einzeln zu stellen und im Konkreten zu beantworten. Was sich so im Konkreten materialisiert, lässt sich als Heterotopie-Miniatur fassen. Der Raum für Abweichung konkretisiert sich auf (mobilen) Endgeräten an unbestimmten Orten.

In der miniaturisierten Abweichungsheterotopie kommt es dementsprechend auch nicht mehr zu kollektiven Abweichungserfahrungen, sondern $\mathrm{zu}$ fragmentierten. So fragmentiert wie die Heterotopie-Miniaturen sind, haben sie jederzeit parallel zu jeder denkbaren anderen Heterotopie auf einem Endgerät Platz - im Falle der so genannten digitalen Phänotypisierung sogar vernetzt mit jedem anderen Bit der genutzten IKT, da hier das gesamte Kommunikationsverhalten von User^innen gescannt und analysiert wird. Die Erscheinungsform der Heterotopie-Miniatur kann verstanden werden als ist das interaktive, Bildpunkte umfassend verknüpfende technische Bild. Hier vergegenständlicht sich die bereits erwähnte Verschränkung von Black Boxes etwa wenn User`innen per E-mail darüber informiert werden, dass ein neues Modul ihrer kognitiv-behavioristischen online-Behandlung zur Verfügung steht, ein entsprechender Link angeklickt wird und im Rahmen des Moduls über weitere Verlinkungen Videos mit therapeutischer Ansprache angesteuert werden können. Gleichzeitig ist es denkbar, dass ein- und dieselbe Oberfläche eines Anbieters von Video-Telefonaten für psychotherapeutische Gespräche und für die Kommunikation mit Freunden oder Familie genutzt wird. Der heterotopische Funktionswandel bezieht sich hier also auf den Kanal. Exemplarisch lässt sich dies am Problem des Schweigens verdeutlichen: Während ein guter Freund einem kurzen Schweigen im Gespräch möglicherweise keine Bedeutung beimisst, könnte dies im Rahmen einer psychotherapeutischen Sitzung eventuell ganz anders gewertet werden. Dies kann brisant werden, weil bestimmte Anbieter Schweigen auf absolute informationelle Stille reduzieren, was beim Gegenüber mitunter den Eindruck der Verbindungsstörung aufkommen lässt. Die Interpretation von Schweigen ist also vom Kanal abhängig.

Die Zugänge zur Miniatur-Heterotopie der digitalisierten Psychiatrie, ihre Öffnungen und Schließungen sind so grundlegend wie die Frage, ob Internet-Zugang, Endgerät und eine Einstellung zur Verfügung stehen, die den Eintritt überhaupt erlauben. Gleichzeitig sind sie so herausfordernd wie das Ablegen des Handtuchs in der Sauna, denn reine Nacktheit mag schon anspruchsvoll sein, wenn man freiwillig sämtliche Daten auf Endgeräten und in den Wolken zum diagnostischen Scan freigibt. Während jedoch das Allerinnerste in der Sauna verborgen bleiben kann, gehört seine Erkundung zum Spektrum der Möglichkeiten der digitalisierten Psychiatrie. Im Gegensatz zum Wundtschen Labor muss es hier nicht bei der Vermessung der Wahrnehmung bleiben, sondern es kann zu umfassenden Verhaltensrekonstruktionen, zu Typisierungen und Diagnosen, zur Vorhersage, Behandlung und Kontrolle der Psyche kommen. 


\section{Fazit}

Räume der psychiatrischen Landschaft fügten sich schon vor ihrer digitalen Erweiterung nicht in einfache Zeit- oder Raumstrukturen ein. Regelmäßigkeit des psychiatrischen Alltags oder unbestimmte Dauer des Aufenthalts im psychiatrischen Anderswo können als exemplarisch für ein Dehnen oder ein Stauchen von Zeit verstanden werden. Die nach Foucault prototypische Abweichungsheterotopie kann vollständig gegenüber dem nicht-psychiatrischen Außen abgeschlossen sein - insbesondere dann, wenn Aufenthalte unfreiwillig stattfinden, wie es etwa im Bereich der forensischen Psychiatrie der Fall ist. Sie kann aber auch gegenüber dem nicht-psychiatrischen Außen weit geöffnet werden, wie dies im Handeln psychiatrischer Ambulanzen geschieht, wo Klienten zu Hause aufgesucht werden. Der psychiatrische Raum kann der Funktion dienen, Teilhabe zu ermöglichen aber auch der Funktion, das nicht-psychiatrische Außen zu verweigern. Der psychiatrische Raum, der sich dem Miteinander zwischen Beschäftigten und Patient*innen verschrieb und der nicht eine Anstalt sein sollte, in die eingesperrt wurde, sondern ein Ort des gemeinsamen Tuns mit flachen Hierarchien zwischen allen, die an diesem Tun beteiligt sind, war vor der Reform der Psychiatrie ein irrealer. Real wurde dieser Raum in Deutschland erst durch sozialpsychiatrische Praktiken seit den späten 1970er Jahren. ${ }^{52}$

Eine theoretische Möglichkeit dieses komplexe Bild aufzufalten und mit Foucault Funktionswandel, Multiplizität, zeitliche Brüche, und Modi der Öffnungen und Schließungen sichtbar zu machen, ist sein Konzept der Heterotopie. Die hier genanten Begriffe sensibilisieren für einen theoretischen Zugang, der auch für Räume der digitalisierten Psychiatrie nutzbar gemacht werden kann. Was im letzten Abschnitt als digital-psychiatrische Miniatur-Heterotopie bezeichnet wurde, ist klein wie ein Smartphone und gleichzeitig so komplex, dass eine nahezu unüberschaubare Vielzahl digitaler Räume mit unterschiedlichsten Funktionen angesteuert werden kann. Das Heterotopie-Konzept kann hier als Ausgangspunkt betrachtet werden, von dem aus methodologisch weiterzuarbeiten wäre. Der Zugang kann helfen zu erkennen, welche unterschiedlich verschränkten Räume eine Miniatur-Heterotopie auf Basis welcher Grundsätze konstituieren. Mit Flusser wäre zu fragen, ob diese verschränkten Räume lediglich als Teil der einer SuperBlack Box zu verstehen wären und ob jede einzelne Bildfläche nur als Feedbacksystem im Sinne einer kybernetischen Steuerung zu verstehen wäre oder ob User^innen selbstbestimmte Änderungen an den Apparateeinstellungen vornehmen können.

52 Vgl. Dörner et al. 2019. 


\section{Literaturverzeichnis}

Bröckling, Ulrich (2008), „Über Feedback. Anatomie einer kommunikativen Schlüsseltechnologie“, in: Michael Hagner u. Erich Hörl (Hgg.), Die Transformation des Humanen. Beiträge zur Kulturgeschichte der Kybernetik, Frankfurt a. M., 326-347.

Bruder, Klaus-Jürgen (1982), Psychologie ohne Bewußtsein. Die Geburt der behavioristischen Sozialtechnologie, Frankfurt a. M.

Castel, Francoise/Castel, Robert/Lovell, Anne (1982), Psychiatrisierung des Alltags. Produktion und Vermarktung von Psychowaren in den USA, Frankfurt a. M.

Canguilhem, Georges (2013 [1943]), Das Normale und das Pathologische, Berlin. - ders. (1943), Le normal et le pathologique, Straßburg. - ders. (1966), Le normal et le pathologique. Erweiterte zweite Ausgabe, Paris.

Chlada, Marvin (2005), Heterotopie und Erfahrung, Aschaffenburg.

DGPPN (2018), Die Psychiatrie und Psychotherapie der Zukunft, Kongress der Deutschen Gesellschaft für Psychiatrie, Psychotherapie und Nervenheilkunde 28. November-1. Dezember, Berlin.

Didi-Huberman, Georges (1997), Erfindung der Hysterie, München.

Dörner, Klaus/Plog, Ursula/Bock, Thomas/Brieger, Peter/Heinz, Andreas/Wendt, Frank (Hgg.) (2019), Irren ist menschlich. Lehrbuch für Psychiatrie und Psychotherapie, Köln.

Eckart, Georg (2010), Kernprobleme in der Geschichte der Psychologie, Wiesbaden.

Fechner, Gustav Theodor (1860), Elemente der Psychophysik, Leipzig.

Floridi, Luciano (2015 [2014]), Die 4. Revolution. Wie die Infosphäre unser Leben verändert, Frankfurt a. M. - ders. (2014), The Fourth Revolution. How the Infosphere is Reshaping Human Reality, Oxford.

Flusser, Vilém (1989), Für eine Philosophie der Fotografie, Göttingen.

Foucault, Michel (2005 [1984]), „Von anderen Räumen“, in: Daniel Deferts u. François Ewald (Hgg.), Michel Foucault. Schriften in vier Bänden. Dits et Ecrits. Band IV, Frankfurt a. M., 931-942. - ders. (1984 [1967]): „Des espaces autres“ (Vortrag im Cercle d'etudes architecturales, 14. März 1967), in: Architecture, Mouvement, Continuite 5, 46-49.

Foucault, Michel (1978 [1977]), „Ein Spiel um die Psychoanalyse. Gespräch mit Angehörigen des Departements de Psychoanalyse der Universität Paris/Vincennes“, in: ders., Dispositive der Macht, Berlin, 118-175. - ders. (1977), „Le jeu de Michel Foucault“, in: Ornicar? Bulletin periodique du champ freudien 1977 (10), 62-93.

Friedrich, Walter/Noack, Klaus-Peter/Bönisch, Siegfried/Bisky, Lothar (Hgg.) (1979), Zur Kritik des Behaviorismus, Köln.

Helmholtz, Hermann von (1856), Handbuch der Physiologischen Optik, Leipzig.

Kittler, Friedrich (1997), „Farben und/oder Maschinen denken“, in: Wolfgang Coy, Georg-Christoph Tholen u. Martin Warnke (Hgg.), HyperKult. Geschichte, Theorie und Kontext digitaler Medien, Basel, 83-97.

Krämer, Sybille (2003), „Erfüllen Medien eine Konstitutionsleistung? Thesen über die Rolle medientheoretischer Erwägungen beim Philosophieren“, in: Stefan Münker, Alexander Roesler u. Mike Sandbothe (Hgg.), Medienphilosophie. Beiträge zur Klärung eines Begriffs, Frankfurt a. M., 78-90.

Krassmann, Susanne (2013), „Monitoring“, in: Ulrich Bröckling, Susanne Krassmann u. Thomas Lemke (Hgg.), Glossar der Gegenwart, Frankfurt a. M., 167-173.

Lamberti, Georg (1995), Wilhelm Maximilian Wundt (1832-1920). Leben, Werk und Persönlichkeit in Bildern und Texten, Bonn.

Latour, Bruno (2002), Die Hoffnung der Pandora. Untersuchungen zur Wirklichkeit der Wissenschaft, Frankfurt a. M. 
Licklider, Joseph Carl Robnett (1973), „Communication and Computers“, in: George A. Miller (Hg.), Communication, Language and Meaning. Psychological Perspectives, New York, 196-207.

Link, Jürgen (2006), Versuch über den Normalismus, Göttingen.

Mills, John A. (1998), Control, New York.

Mol, Annemarie (2017), „Krankheit tun“, in: Susanne Bauer, Torsten Heinemann u. Thomas Lemke (Hgg.), Science and Technology Studies. Klassische Positionen und aktuelle Perspektiven, Frankfurt a. M., 429-467.

Mucic, Davor/Hilty, Donald M. (2016), e-Mental Health, Heidelberg.

Rechenberger, Helmut (1994), Hermann von Helmholtz, Bilder seines Lebens und Wirkens, Weinheim.

Regener, Susanne (1999), Fotografische Erfassung, München.

Regener, Susanne (2010), Visuelle Gewalt. Menschenbilder aus der Psychiatrie des 20. Jahrhunderts, München.

Rheingold, Howard (1985), Tools for Thought, New York.

Rosenblueth, Arturo/Wiener, Norbert/Bigelow, Julian (1943), „Behavior, Purpose and Teleology“, in: Philosophy of Science 10 (1), 18-24.

Schumann, Friedrich (2004), Bericht über den 1. Kongress für experimentelle Psychologie in Gießen vom 18. bis 21. April 1904, Göttingen.

Stingelin, Martin (1988), „En face et en profil. Der identifizierende Blick von Polizei und Psychiatrie“, in: Sprengel Museum (Hg.), Fotovision, Projekt Fotografie nach 150 Jahren, Hannover, 181-191.

Turkle, Sherry (1984), The Second Self. Computers and the Human Spirit, New York.

Watson, John B. (1968 [1923]), Behaviorismus, Köln. - ders. (1923), Psychology from the Standpoint of a Behaviorist, Philadelphia.

Wiener, Norbert (1963 [1948]), Kybernetik. Regelung und Nachrichtenübertragung im Lebewesen und in der Maschine, Düsseldorf. - ders. (1948), Cybernetics. Or control and communication in the animal and the machine, Cambridge (MA).

Wundt, Wilhelm (1983a [1862]), „Beiträge zur Theorie der Sinneswahrnehmung. Einleitung: Über die Methode in der Psychologie“, in: ders., Psychologische Studien. Bd.1, München, 1-22.

Wundt, Wilhelm (1983b), „Das Institut für experimentelle Psychologie zu Leipzig“, in: ders., Psychologische Studien. Bd. 4, München, 511-525.

Ziemann, Andreas (2017), Das Bordell: Historische und soziologische Beobachtungen, Weilerswist.

Lillemose, Jacob/Kryger, Mathias (2015), „The (Re)invention of Cyberspace“, in: Kunstkritikk. Nordic Art Review, kunstkritikk.com/the-reinvention-of-cyberspace (Stand: 26.8.2019).

The Abilify Mycite System (2019), www.abilifymycite.com (Stand: 26.8.2019).

WHO (2016), Global diffusion of e-Health: Making universal health coverage achievable, Genf. Auch online verfügbar: https://www.who.int/goe/publications/global_diffusion/en/ (Stand: 26.8.2019). 